\title{
Liénard, F. et Zlitni, S. (dir.), Regards croisés sur la communication et la trace numériques
}

Fabien Wille

\section{(2) OpenEdition}

\section{Journals}

Édition électronique

URL : https://journals.openedition.org/edc/12159

DOI : $10.4000 /$ edc. 12159

ISSN : 2101-0366

Éditeur

Université de Lille

Édition imprimée

Date de publication : 23 juillet 2021

Pagination : 191-194

ISBN : 978-2-917562-25-3

ISSN : $1270-6841$

Référence électronique

Fabien Wille, "Liénard, F. et Zlitni, S. (dir.), Regards croisés sur la communication et la trace numériques », Études de communication [En ligne], 56 | 2021, mis en ligne le 23 juillet 2021, consulté le 02 janvier 2023. URL : http://journals.openedition.org/edc/12159 ; DOI : https://doi.org/10.4000/edc.12159

Ce document a été généré automatiquement le 2 janvier 2023.

Tous droits réservés 


\title{
Liénard, F. et Zlitni, S. (dir.), Regards croisés sur la communication et la trace numériques
}

\author{
Fabien Wille
}

\section{RÉFÉRENCE}

Liénard, F. et Zlitni, S. (dir.) (2020). Regards croisés sur la communication et la trace numériques. Presses universitaires de Rouen et du Havre.

\section{Fabien Liénard et Sami Zlitni (dir.), Regards croisés sur la communication et la trace numérique ${ }^{1}$}

1 Comme il est indiqué sur la quatrième de couverture, « ce volume est le premier d'une collection consacrée à la trace numérique ». Le paradigme de l'homme-trace développé par les chercheurs en SIC de l'université Le Havre Normandie, est issu d'une proposition anthropologique de Béatrice Galinon-Mélénec qui considère que «l'homme est à la fois un producteur de traces et le produit de traces ou, plus précisément, de signes traces » (p. 11).

Cet ouvrage, qui se veut fondateur, se compose d'un avant-propos qui pose le cadre général, «Du paradigme de l'Homme-trace à la trace numérique ». Malgré quelques tentatives, amplement nourries des travaux de Jeanneret, toute imposition d'une définition, unique, légitime et univoque, semble illusoire. Ainsi considérée comme la trace d'une pratique sociale particulière dans une culture, un lieu et une temporalité spécifiques, la complexité réside dans la circulation des représentations qui lui sont attachées dans l'espace médiatique. Ces analyses se trouvent ainsi affectées par l'irruption des technologies du numérique. L'avant-propos, positionnant ainsi les travaux du laboratoire sur cette question, est suivi de huit chapitres. Si les deux 
premiers chapitres posent le cadre conceptuel, le reste de l'ouvrage est davantage consacré à l'analyse de différents terrains.

3 Le chapitre 1, «Pour une ichnologie générale à l'heure du numérique », permet ainsi à Béatrice Galinon-Mélénec, au fil des 46 pages, de calibrer le paradigme proposé au moyen d'une bibliographie abondante et selon quatre grands chapitres sur le positionnement épistémologique, la trace en contexte numérique, les règles du numérique conçues par l'Humain, et la trace numérique analysée en tant que lettrure, avant de conclure. Il est alors précisé que la préférence sémiotique du lecteur est actrice de l'interprétation de la trace. Cette trace est ainsi appréhendée comme un ensemble de " nœuds de conséquences » difficile à déconstruire et impossible à prévoir. L'auteure fait ainsi le constat d'une trace qui nous échappe, nous empêchant de clore définitivement sa signification. L'éthique apparait alors comme un repère au regard d'un contexte interprétatif qui constitue un obstacle à toute tentative de définition stable de la trace.

4 Dans le chapitre 2, Alexandra Saemmer poursuit le travail de contextualisation. "Prolégomènes à une sémiotique sociale» nous invite, face à une production culturelle, à engager une critique des stratégies de communication, empruntant à Nolwenn Tréhondart le concept d'«introspection idéologique » qui vise à la fois à la conscientisation de l'action de la matérialité des signes et également celle des points de vue, systèmes de croyances et stéréotypes, dans le processus interprétatif.

5 L'ouvrage propose ensuite une diversité de terrains d'analyse à partir du chapitre 3, "Trace expérientielles et formes de vie: une sémiogenèse d'une séquence expérientielle à la cité du vin (Bordeaux)». Jean-Jacques Boutaud se donne pour ambition d'ouvrir une perspective post-expérientielle, se nourrissant des apports sur le «modal ", et ainsi entrevoir la production et la reconnaissance de traces comme «la manifestation de styles et de formes de vie qui cherchent en permanence les moyens de s'exprimer, dans la pluralité des modes d'être, de l'expérience à l'existence ». Le chapitre 4 propose "[U]ne déconstruction sociologique du mythe des digital natives et de la génération $Y$ » par Pascal Lardellier. Pour l'auteur, la " génération $Y$ » constitue un mythe, déconstruisant ainsi toute assignation autour des digital natives. Dans le chapitre 5, « Bibliothèque numériques interopérables et data », Fabrice Papy analyse les promesses sociotechniques anthropocentrées pour la "société de l'information». Il démontre que les potentialités technologiques et les promesses qui lui sont attachées ne préfigurent en rien des usages effectifs d'un public aux profils informationnels et techniques variés. Dans le chapitre suivant, «Les réseaux sociaux numériques et marques : le spectacle publicitaire continu ", Karine Berthelot-Guiet convoque in fine un concept qu'elle a développé en 2013 autour de la notion de "publicitarité », structurée par la maîtrise de la communication et par le pouvoir de la représentation. Il apparaît ainsi que les marques réactivent au sein des réseaux sociaux ce principe.

6 Pour Bernard Idelson et Élodie Lauret, auteurs du chapitre 7, "L'espace médiatique réunionnais: entre transformations numériques et habitus éditoriaux", si le développement du numérique s'accompagne d'une transformation des supports, celleci confirme quatre aspects du journalisme de marché décliné selon les particularismes insulaires: les rubriques et une perte d'autonomie qui valorisent les flux, tout en préservant sa tradition d'un journalisme d'opinion et la conservation d'un habitus éditorial de débats et de commentaires. Enfin dans le chapitre 8, «L'iconisation du discours dans les urbanitécrans: applications mobiles et photographies déictiques ", 
Marie-Anne Paveau montre comment les pratiques de numérisation ramènent les usages de la ville dans la sphère du " me, myself and I » et comment, parallèlement, les traits et fonctionnements des nouvelles modalités des urbanitécrans impactent les théories et méthodes des disciplines qui s'occupent de ces objets.

7 Il n'y a pas de conclusion générale de l'ouvrage. On peut néanmoins apprécier cette contribution à une réflexion menée depuis dix ans sur la question des traces numériques. Elle s'opère par une double inscription du propos, d'une part grâce à une formalisation de son ancrage théorique et d'autre part, au moyen de différentes études de terrains.

9 Univ. Lille, ULR 4073 - GERiiCO, F-59000 Lille, France

fabien.wille@univ-lille.fr

\section{NOTES}

1. Liénard, F. et Zlitni, S. (dir.) (2020). Regards croisés sur la communication et la trace numériques. Presses universitaires de Rouen et du Havre.

\section{AUTEURS}

\section{FABIEN WILLE}

Univ. Lille, ULR 4073 - GERiiCO, F-59000 Lille, France, fabien.wille@univ-lille.fr 